\title{
A pré-história dos buracos negros
}

\author{
Before black holes
}

\author{
Carla R. Almeida*10 \\ ${ }^{1}$ Max Planck Institute for Gravitational Physics, Historical Epistemoloy of the Final Theory Program, Berlin, Germany.
}

Recebido em 14 de Maio de 2020. Revisado em 19 de Junho de 2020. Aceito em 24 de Junho de 2020.

\begin{abstract}
Buracos negros são provavelmente a mais estranha consequência da relatividade geral. Eles são objetos super densos envolvidos por uma membrana unidirecional que escondem uma singularidade em seu interior e aprisionam qualquer matéria que se arrisca a adentrá-lo, incluindo a luz. A história da descoberta desses corpos enigmáticos se inicia com a descoberta da primeira solução das equações de Einstein em 1916, mas a publicação que os introduziu foi lançada apenas em 1939. Este artigo apresenta a sequência de fatos da fase inicial de pesquisas que posteriormente levaram ao surgimento do conceito de buracos negros, apresentando uma análise social, filosófica e política sobre o tema.
\end{abstract}

Palavras-chave: buracos negros, história da física, Oppenheimer, hipótese do equilíbrio, estrelas colapsadas.

Black holes are arguably the most counter-intuitive consequence of general relativity. They are super dense objects surrounded by unidirectional membranes, each hiding a singularity inside. A black hole traps everything that enters it, including light. The history of the discovery of those enigmatic objects starts in 1916, with the first solution to Einstein's Equations for the gravitational field of a massive spherical-symmetric dimensionless body. But the publication that introduced them appeared only in 1939. This paper presents the historical events of the initial phase of research that led to the rise of the black holes as a concept. It presents a social, philosophical, and political analysis of the subject.

Keywords: black holes, history of physics, Oppenheimer, equilibrium hypothesis, collapsed objects.

\section{Introdução}

Nos últimos cinco anos, duas das maiores conquistas do mundo moderno envolveram buracos negros: a primeira detecção das ondas gravitacionais em 2015 pelo time do LIGO $^{1}$ produzidas por um sistema binário de buracos negros, que resultou no Prêmio Nobel para Barry C. Barish e Kip S. Thorne; e a publicação da imagem de um buraco negro em março de 2019 pelos pesquisadores do Telescópio Event Horizon, construída com uma técnica inovadora - a combinação de técnicas de machine learning com dados observacionais para a produção de imagens mais precisas. Buracos negros são objetos super densos que produzem um forte campo gravitacional, distorcendo o espaço-tempo à sua volta, criando uma região de onde nada pode escapar. O nome reflete as principais propriedades que definem o conceito. Buraco se refere à curvatura que este impõe ao espaço-tempo, que permite a entrada, mas não a saída de partículas. Este é delimitado por uma membrana fictícia, o horizonte de eventos. Negro remete ao fato de que nem a luz consegue passar da região interna para a externa e, então, o buraco negro

\footnotetext{
*Endereço de correspondência: cralmeida00@gmail.com

1 LIGO é a sigla em inglês para Observatório de Ondas Gravitacionais por Interferômetros a Laser (Laser Interferometer Gravitational-Waves Observatory).
}

é invisível contra o fundo preto, podendo ser observado apenas pela ação do seu campo gravitacional.

Primeiro descobertos teoricamente, buracos negros foram previstos muito antes de serem observados. Por esta razão, o caminho histórico das pesquisas desses objetos apresenta muita controvérsia e ceticismo. Historicamente, podemos identificar quatro fases distintas no desenvolvimento das pesquisas em buracos negros, cada uma caracterizada pelo status da ideia. Na primeira fase, a ideia de um corpo massivo comprimido em um pequeno volume, além do que hoje chamamos de horizonte de eventos, era absurda. Em seguida, a aceitação das consequências teóricas da relatividade geral (RG) tornou a existência destes objetos plausível. Com as descobertas astronômicas dos quasares e pulsares e a ascensão da astrofísica relativística, a ideia se tornou amplamente aceita. Foi também nesta fase que o termo 'buraco negro' se popularizou, tanto dentro da comunidade científica, quanto na consciência popular. Por fim, a incessante busca por um buraco negro no espaço atinge seu auge com as observações do LIGO e do Telescópio do Event Horizon, comprovando de uma vez por todas a existência destes objetos. Embora seja difícil precisar exatamente o começo e fim de cada fase, é possível apontar eventos particulares que ajudaram na transição de uma para outra. Este artigo focará na transição entre os períodos em que a ideia era considerada absurda e de quando ela se 
tornou plausível. O pivô dessa mudança foi um artigo de 1939 escrito por John Robert Oppenheimer e Hartland Snyder, Sobre a Contração Gravitacional Continuada.

Para entender a resistência inicial à ideia e a subsequente mudança de mentalidade, este trabalho apresenta uma análise do contexto filosófico e sociológico das décadas de 1920 a 1940. Este período foi caracterizado pela falta de evidências a favor da existência de buracos negros, com apenas alguns avanços teóricos apontando nesta direção. A nomenclatura acompanhou este avanço. As primeiras soluções da equação de Einstein se referiam a ele como Massenpuktes, ou massas puntuais. Em 1930, a palavra colapso foi introduzida num contexto astrofísico e então surgiu o termo objetos completamente gravitacionalmente colapsados. O familiar nome pelo qual conhecemos estes objetos hoje só apareceria décadas após, popularizado pelo físico John A. Wheeler depois de 1967.

Este artigo apresenta também uma discussão sobre como a Segunda Guerra Mundial (1939-1945) interveio nessa história. Ele está dividido da seguinte forma: a próxima seção apresenta um resumo cronológico dos avanços em física que levaram ao artigo seminal de Oppenheimer e Snyder, explicando os conceitos e a evolução científica das pesquisas. Em seguida, o contexto social e filosófico deste período é revisado. Por fim, há uma discussão sobre as consequências da Segunda Guerra Mundial para as pesquisas de larga-estrutura, como astronomia, astrofísica, cosmologia e relatividade geral. Este trabalho é baseado no artigo [1].

\section{Antes dos Buracos Negros: O Nascimento da Relatividade Geral e Desenvolvimentos em Astrofísica}

O físico alemão Karl Schwarzschild obteve a primeira solução das Equações de Einstein em 1916 [2,3], menos de um ano após a publicação seminal que marca o nascimento da relatividade geral. Embora essa solução descreva um buraco negro, tal consequência só foi entendida décadas depois. Na época, a ideia de um objeto massivo sem dimensões era contra-intuitiva e não tinha suporte observacional. De qualquer forma, estes artigos marcam o início das investigações sobre este tema. A solução de Schwarzschild, como ficou conhecida, descreve o campo gravitacional de uma partícula pontual massiva (sem carga e sem rotação) e apresenta em sua formulação duas singularidades: uma na origem do sistema de coordenadas, onde a massa se concentraria, e uma radial, o raio de Schwarzschild, que na época acreditava-se delimitar uma região para qual a solução não mais seria válida.

Como foi inicialmente idealizada, a solução de Schwarzschild está escrita nas coordenadas de um observador distante. Alguém afastado da origem que observa uma partícula em trajetória radial em direção ao centro percebe que esta demoraria um tempo infinito para atingir o raio de Schwarzschild, nunca cruzando-o. Foi apenas em 1933 que o físico belga George Lemaître demonstrou que este raio não era uma barreira intransponível [4], isto é, ele é uma singularidade aparente. Considerando um observador comóvel, que se move junto com o objeto em direção ao centro, pode-se não apenas alcançar o raio de Schwarzschild, como também ultrapassá-lo em tempo finito. Mais tarde, nesta mesma década, Howard P. Robertson percebeu que, embora seja preciso um tempo finito para chegar ao interior desse raio, trajetórias na direção oposta, partindo de dentro, nunca cruzariam essa barreira de volta. Aquilo que adentra o raio de Schwarzschild nunca escapa. Embora Robertson tenha obtido estes resultados na década de 1930, eles só foram publicados postumamente [5].

A percepção de que o raio de Schwarzschild é uma singularidade aparente não era suficiente para concluir que objetos compactos como buracos negros poderiam existir. A solução de Schwarzschild provê uma fórmula para o campo gravitacional de um objeto esfericamente simétrico, como uma estrela ou uma partícula, por exemplo. Porém, há outras forças que atuam sobre esses corpos esféricos que poderiam contrabalancear à gravitacional e impedir a existência de objetos com raios menores do que o de Schwarzschild. Como Lemaître mesmo observou, "apenas as forças nucleares subatômicas parecem ser capazes de parar a contração do universo, quando o raio dele é reduzido à dimensões do sistema solar" 2 [4, p.679] Em 1920, o objeto mais denso conhecido era uma anã branca, e a sua densidade estimada já era muito maior do que as observadas na Terra. Na tentativa de explicar a formação de tais corpos, o astrônomo britânico Arthur S. Eddington apresentou uma teoria detalhando a constituição interna de estrelas, que se tornou o modelo padrão para estrelas nas décadas seguintes, até a descoberta da fusão e fissão nucleares em 1939. Neste modelo, estrelas são formações esféricas de um gás perfeito e "a pressão elétrica, a pressão gasosa e a pressão de radiação" [6, p.321] a manteriam em equilíbrio com a força gravitacional.

Neste contexto, a hipótese de Schwarzschild de um corpo pontual massivo era considerada uma idealização teórica (inclusive pelo próprio Schwarzschild). Em realidade, acreditava-se não existir um objeto com dimensões menores do que o raio de Schwarzschild e, portanto, a região problemática, o raio singular, estaria para sempre escondida no interior das estrelas, onde a solução não é mais válida. Essa discussão se deu paralelamente ao advento da mecânica quântica (MQ). Não demorou muito até que os então novos métodos de estatística quântica fossem usados para investigar o interior estelar. Em 1930, Edward Milne foi um dos primeiros a questionar o modelo estelar de Eddington [7] e, mais tarde, dois jovens físicos, Subrahmanyan Chandrasekhar e Lev Landau, seguiram seus passos. Eles encontraram, respectivamente, um limite máximo para as massas de anãs

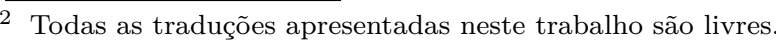


brancas [8] e estrelas de nêutron [9] ${ }^{3}$ Embora estes resultados implicassem no colapso gravitacional de estrelas super massivas, Chandrasekhar e Landau não ousaram sugerir essa possibilidade. Ambos argumentaram, a princípio, que as conhecidas leis da física não valeriam para corpos de massas grandes. Essa conclusão tinha o único propósito de evitar a contração destes objetos até um ponto singular, sob efeito do próprio campo gravitacional.

Em seu trabalho, Landau admitiu que para densidades maiores do que a considerada, uma estrela colapsaria na origem. Ele comentou que "em toda a teoria quântica não há uma causa que previna o sistema de colapsar até um ponto. Mas, como na realidade essas massas existem quietas como estrelas e não demonstram essa tendência ridícula, devemos concluir que estrelas mais pesadas que 1,5 massas solares certamente possuem regiões onde as leis da mecânica quântica (e, portanto, da mecânica estatística) são violadas". [9, p.272] Chandrasekhar, por outro lado, foi mais cauteloso ao duvidar dos resultados teóricos. Num segundo artigo publicado em 1935 e apresentado numa reunião da Sociedade Real de Londres, ele deixou em aberto o destino das estrelas mais pesadas [10], evitando afirmações como a de Landau de que as leis físicas deveriam falhar nesses casos. Durante o processo de pesquisa, Chandrasekhar consultou Eddington, que era professor sênior de Cambridge, algumas vezes. Eddington inclusive emprestou sua calculadora eletrônica para que o então doutorando terminasse seus cálculos, sem nunca comentar sobre eles. Foi, então, um enorme choque para Chandrasekhar quando Eddington atacou o seu trabalho, naquele mesmo dia. Eddington era completamente contrário à ideia de colapso gravitacional e apontou que o resultado de Chandrasekhar indica que isto aconteceria para estrelas supermassivas. Ele concluiu sua palestra professando: "Eu acho que deve haver algo na natureza que previna a estrela de se comportar dessa forma absurda!" [11, p.160] Arrasado, Chandrasekhar só voltou a pesquisar sobre o assunto décadas depois. Naquela época, a autoridade de Eddington e a estranheza do resultado, que ainda não tinha um suporte observacional, foram o suficiente para desacreditar o trabalho de Chandrasekhar. O primeiro artigo a argumentar em favor ao colapso gravitacional veio de um lugar muito distante do lar científico de Eddington em Cambridge, Inglaterra.

Sobre a contração gravitacional continuada, de J. Robert Oppenheimer e Hartland Snyder, pesquisadores de Berkeley na Califórnia, EUA, foi publicado em Setembro de 1939 [12]. Eles são pioneiros em aplicar relatividade geral nesse contexto astrofísico, usando a descrição relativística da termodinâmica de Richard Tolman [13] para re-analisar os resultados de Landau. Assim, eles concluem que estrelas supermassivas se contraem indefi-

\footnotetext{
3 Em verdade, o resultado de Landau precede à descoberta da partícula nêutron e estrelas de nêutron foram observadas apenas 35 anos depois. A densidade que ele considera é equivalente a de uma estrela de nêutron, embora ele não soubesse disso na época.
}

nidamente, ultrapassando o raio de Schwarzschild, até o centro de coordenadas, em tempo finito. Embora todas as componentes teóricas necessárias para deduzir que o colapso gravitacional completo de uma estrela poderia acontecer estivessem disponíveis desde 1934, o trabalho de Oppenheimer e Snyder foi ousado em desafiar a crença da época, de que estrelas deveriam permanecer em equilíbrio. Demoraria mais 20 anos até que a comunidade científica levasse esse resultado a sério.

\section{Filosofia: Estrelas Devem Permanecer em Equilíbrio}

Para entender porque a existência de buracos negros era considerada absurda nas primeiras duas décadas após o nascimento da relatividade geral, é necessário analisar também o contexto filosófico da época. Já comentamos sobre a falta de evidências observacionais e sobre a impossibilidade de testar tal hipótese. Mas a negação das evidências teóricas que apareceram no início da década de 1930 é de característica mais filosófica do que científica. As visões empiristas e instrumentalistas do final do século XIX até meados do século XX são componentes chave para a negação do colapso estelar. A característica mais poderosa do empirismo é o papel dos sentidos na construção do conhecimento. Em outras palavras, além da necessidade de evidências, a percepção sensorial era parte importante para a formulação de uma teoria física. O colapso gravitacional completo desafiava essa filosofia, implicando a existência de uma região no universo fora do alcance da observação. Um domínio fechado em si mesmo, fora do escopo de possibilidades de medidas.

Depois de 1920, o trabalho sobre a constituição das estrelas e o carisma natural para comunicação científica transformaram Arthur S. Eddington em um dos mais influentes físicos da época. Ele também foi o maior antagonista da ideia de colapso gravitacional. Eddington era um físico brilhante e entendia bem as consequências deste, descrevendo com detalhes impressionantes, num experimento mental, a contração gravitacional, em seu livro A Constituição Interna das Estrelas, de 1926. Ele concluiu que "a massa produziria tanta curvatura da métrica do espaço-tempo que o espaço se fecharia ao redor da estrela, nos deixando do lado de fora (i.e., em lugar nenhum)". [14, p.6] Este fato ia de encontro às visões filosóficas dele. Ele escreveu mais tarde, em seu livro Estrelas e Átomos de 1929, "Eu devo enfatizar que todo nosso conhecimento físico é baseado em medidas e que o mundo físico consiste, por assim dizer, em grupos de medidas em um fundo sombreado que reside fora da física. Portanto, ao conceber um mundo cuja existência é independente de medições, eu ultrapasso os limites do qual chamo de realidade física". [15, p.152]

A filosofia de Eddington sumariza a visão geral daquele período. E, como uma autoridade reconhecida da área, ele inadvertidamente impôs a crença de que estrelas permaneceriam em equilíbrio hidrostático e que as forças 
nucleares internas de um corpo contrabalanceariam a gravitacional. Sem evidências observacionais, a comunidade científica aceitou essa hipótese do equilíbrio. Como Landau mesmo disse, estrelas não apresentavam essas "tendências ridículas" de se contraírem até um ponto de densidade infinita.

A oposição de Einstein, por outro lado, era de natureza mais geométrica, contra a ideia da existência de singularidades no espaço-tempo. Ele considerava isso inaceitável, "pois uma singularidade traz tanta arbitrariedade para a teoria que de fato a nulifica." [16, p.73] As motivações filosóficas também iam além da ideia de buracos negros. A relatividade geral em si estava passando por um período de águas-rasas [17], caracterizado pela diminuição de publicações e, em geral, pouco progresso. A teoria da gravitação de Einstein ainda estava em sua infância, tentando provar a si mesma para a comunidade científica. Depois da expedição de Sobral em 1919 para medir a deflexão da luz sob a influência do campo gravitacional do Sol, a RG ganhou mais atenção, mas também mais críticos. Em meados da década de 1920, ela mergulhou num período de estagnação que durou até meados da década 1950. Em face desse atraso, os detalhes da teoria que escondiam o buraco negro ficaram perdidos por muito tempo.

A implementação desse postulado não-escrito de que estrelas permaneceriam em equilíbrio levou os físicos a se perguntarem 'como' evitar o colapso gravitacional, ao invés de 'por que' ele acontece. As pesquisas, então, focaram-se em torno desta primeira questão. Eddington serviu como o maior propagador da hipótese do equilíbrio. Chandrasekhar comenta para seu biógrafo: "Eddington poderia ter feito. Quando digo que ele poderia ter feito, eu não estou apenas especulando. Estava completamente dentro da filosofia que caracteriza seu trabalho sobre a constituição das estrelas. E se Eddington tivesse feito, ele seria hoje considerado o maior astrônomo teórico do século, porque ele teria predito e falado sobre estrelas colapsadas de um ponto de vista completamente relativístico. Foram precisos 30 anos de espera para isso acontecer". [18, p.350]

\section{Califórnia: O Subúrbio da Comunidade Científica}

Para entender a mudança epistemológica que levou Oppenheimer a desafiar a hipótese do equilíbrio, afirmando que o colapso gravitacional era plausível, é necessário uma análise sociológica da comunidade científica da época. Na primeira metade do século XX, a Europa era o centro da produção de conhecimento e discussões filosóficas mundial. Foi o berço das mais revolucionárias teorias do século: relatividade geral e mecânica quântica. Ambas inspiraram muitas disputas filosóficas e impulsionaram o surgimento de grupos como o Círculo de Viena de positivismo lógico. A Universidade de Cambridge na Inglaterra, a instituição anfitriã de Arthur Eddington, referência para pesquisas astrofísicas e astronômicas, estava imersa neste contexto.

O período de águas rasas da relatividade geral coincide com a prosperidade da teoria quântica na Europa. Deste ponto de vista, parece natural que os pesquisadores de Cambridge se orientassem mais em direção ao boom quântico do que à abstrata relatividade geral. Do outro lado do Atlântico, no entanto, as atividades do Observatório de Monte Wilson abriram muitas oportunidades para astrônomos se instalarem na Califórnia. Dentre eles estava Edwin Hubble. As medições feitas por ele em 1929 [19] concordavam com o modelo cosmológico de Lemaître para um universo em expansão. Dando ênfase em cosmologia ao invés de astrofísica, a comunidade californiana foi mais receptiva à relatividade geral, um fato que é refletido nos trabalhos de Richard Tolman e Fritz Zwicky, no Instituto de Tecnologia da Califórnia (Caltech), e de J. Robert Oppenheimer, em Berkeley.

A comunidade californiana não apenas seguiu em uma direção diferente em termos das influências teóricas, mas também filosóficas. Críticas ao empirismo filosófico, especialmente da visão de Eddington, encontraram uma casa no oeste dos Estados Unidos. Esse fato é exemplificado pela amizade entre Tolman e o físico e filósofo natural Herbert Dingle, um crítico ferrenho da filosofia de Eddington. Dingle passou um ano no Caltech em 1937, onde ele e Tolman desfrutaram de várias discussões. Suas críticas às autoridades de Eddington, Einstein e Milne $[20,21]$ provavelmente deixaram uma impressão forte em Tolman, que frequentemente discutia com Oppenheimer sobre os avanços da cosmologia e astrofísica [22].

O desdém de Eddington pelos resultados de Chandrasekhar no encontro da Sociedade Real em 1935, seguido da publicação do artigo de Oppenheimer e Snyder em 1939, sugere que o fator social teve um grande papel na rejeição da ideia do colapso gravitacional. É um indício da influência da comunidade científica e de seu conservadorismo para com ideias inovadoras. A hipótese do equilíbrio foi amplamente aceita na Europa e, em contraste, a comunidade suburbana da Califórnia, a casa científica de Oppenheimer e Snyder na época, estava mais aberta às novidades. Não foi a última vez que os "hippies" Californianos subverteriam as expectativas, "salvando a física" 4

\section{A Segunda Guerra Mundial: O Universo Parou}

Sobre a contração gravitacional continuada foi publicada na edição de $1^{\circ}$ de Setembro de 1939 da revista Physical Review. Embora esse artigo sugira que o colapso é plausível, ele não resolveu todos os problemas. Naturalmente, é preciso mais do que evidências teóricas para aceitar uma conclusão tão contra-intuitiva, mas o artigo de Oppenheimer e Snyder deveria ser suficiente para instigar

\footnotetext{
4 Veja Como os Hippies Salvaram a Física, de David Kaiser [23].
} 
mais investigações sobre o assunto. No entanto, ele foi amplamente ignorado por quase duas décadas.

No mesmo dia em que esse trabalho revolucionário foi publicado, o exército Nazista alemão invadiu a Polônia, dando início à Segunda Guerra Mundial. O mundo se encontrava num contexto político ímpar e os esforços de guerra foram priorizados. Esse foi um período caracterizado pela abundância de investimentos governamentais em pesquisas físicas, que mudou permanentemente o cenário científico [24]. Muitos físicos foram recrutados para trabalhar em projetos militares, incluindo Oppenheimer, que se tornou o líder do Projeto Manhattan, responsável pelo desenvolvimento da bomba atômica americana.

Durante a guerra, pesquisas sobre o cosmo e seus mistérios não eram prioridade. A maioria dos astrônomos, astrofísicos e cosmólogos da época dedicaram-se à investigação de uma física mais imediatamente aplicável, como a nuclear, computacional e tecnologias de rádio. Esse fato não necessariamente indica um apoio à guerra; essa priorização foi devida ao fluxo de investimentos. A maior contribuição à física de largas-estruturas depois do início da guerra veio após o seu final, em 1946: um artigo sobre a síntese dos elementos químicos no interior dos núcleos estelares, por Fred Hoyle, um dos pilares do atual Modelo Padrão da Cosmologia.

A renascença da relatividade geral nos anos 1950 [25] fez ressurgir o interesse pela solução de Schwarzschild, o que eventualmente ajudou com a aceitação mais ampla da ideia de que buracos negros existem. No entanto, o trabalho de Oppenheimer continuou esquecido. Outros fatores podem ser apontados para explicar porque a comunidade científica o ignorou por tanto tempo. No aspecto político, a Segunda Guerra causou uma gigantesca mudança de foco nas pesquisas físicas. Sobre as investigações, não havia uma aplicação imediata dos resultados de Oppenheimer. De um ponto de vista sociológico, a falta de interesse do próprio Oppenheimer em divulgar seu trabalho pode ter sido um importante motivo. Ele desistiu do tema [22].

Na década de 1960, novos avanços teóricos, simulações computacionais e a descoberta observacional de objetos mais densos, os pulsares e os quasares, impulsionaram as pesquisas sobre o tema. A existência de buracos negros foi finalmente aceita. Era uma nova geração de físicos, com uma nova filosofia da ciência. Em 1969, Stephen Hawking e Roger Penrose provaram que singularidades no espaço-tempo são inevitáveis [26]. Em 1971, Remo Ruffini e John Wheeler apresentaram os buracos negros para uma audiência mais ampla [27], sedimentando o conceito no imaginário popular. Novas propriedades foram descobertas nas décadas seguintes e, finalmente, uma imagem foi liberada em março de 2019. Novos tempos para a física dos buracos negros.

\section{Conclusão}

Uma análise retrospectiva do desenvolvimento histórico que levou à descoberta teórica dos buracos negros revela um caminho não-linear de progresso, com obstáculos filosóficos e sociológicos a serem vencidos. Nessa fase inicial de pesquisas, houve uma transição epistemológica que levou a comunidade científica a considerar a plausibilidade da existência de objetos completamente gravitacionalmente colapsados, uma ideia que mais tarde se desenvolveria para o conceito de buracos negros.

No começo dos anos 1920, não havia evidência observacional que suportasse a teoria e havia poucas indicações teóricas em favor à existência de objetos super densos. Neste contexto, é compreensível que a hipótese de que estrelas permaneceriam em equilíbrio hidrostático fosse considerada, nunca atingindo densidades maiores. No entanto, mesmo quando os avanços teóricos começaram a indicar que tais objetos existiam, no começo dos anos 1930, a hipótese do equilíbrio permaneceu inabalável por quase três décadas.

Durante a primeira metade do século XX, a comunidade científica estava centrada na Europa. A distância física entre a Califórnia e a Europa pode ser um fator importante para entender porque os pesquisadores americanos foram menos influenciados pelas tradições científicas européias, criando um ambiente mais favorável à especulação. $\mathrm{O}$ caso dos objetos completamente gravitacionalmente colapsados é um indicador dessa hipótese. Oppenheimer e colaboradores desafiaram a filosofia em evidência na época, não apenas usando relatividade geral em problemas astrofísicos, mas também predizendo a contração continuada de estrelas super massivas, assegurando a plausibilidade do fenômeno hoje conhecido como buraco negro.

O fato do trabalho de Oppenheimer não ter sido reconhecido por muitas décadas é um indicativo do quão problemático esse resultado era. A catastrófica Segunda Guerra Mundial desviou ainda mais a atenção deste resultado. Com isso em mente, a evolução das pesquisas em buracos negros é um estudo de caso histórico interessante para entender como aspectos políticos e sociológicos podem interferir em pesquisas científicas, abrindo uma conversa sobre a importância da filosofia da ciência para seu progresso.

\section{Referências}

[1] C.R. Almeida, The European Physical Journal H, 2102 (2020).

[2] K. Schwarzschild, Sitzungsberichte der königlich Preussischen Akademie der Wissenschaften zu Berlin, 186 (1916).

[3] K. Schwarzschild, Sitzungsberichte der königlich Preussischen Akademie der Wissenschaften zu Berlin 24, 424 (1916).

[4] A.G. Lemaître, General Relativity and Gravitation 29, 641 (1997). 
[5] H.P. Robertson e T.W. Noonan, Relativity and Cosmology (W. B. Saunders, Philadelphia, 1968).

[6] A.S. Eddington, Monthly Notices of the Royal Astronomical Society 5, 308 (1924).

[7] E.A. Milne, Monthly Notices of the Royal Astronomical Society 91, 4 (1930).

[8] S. Chandrasekhar, Monthly Notices of the Royal Astronomical Society 3,456 (1931).

[9] L. Landau, en Neutron Stars, Black Holes and Binary X-Ray Sources, editado por H. Gursky and R. Ruffini (Springer, Dordrecht, 1975).

[10] S. Chandrasekhar, Monthly Notices of the Royal Astronomical Society 3, 207 (1935).

[11] K.S. Thorne, Black Holes 83 Time Warps: Einstein's Outrageous Legacy (W.W. Norton \& Company, New York, 1994).

[12] J.R. Oppenheimer e H. Snyder, Physical Review 5, 455 (1939).

[13] R. Tolman, Relativity, Thermodynamics and Cosmology (Dover Publications, New York, 1987).

[14] A.S. Eddington, On the Internal Constitution of Stars (Cambridge University Press, Cambridge, 1926).

[15] A.S. Eddington, The Nature of Physical World (Cambridge University Press, Cambridge, 1929).

[16] A. Einstein e N. Rosen, Phys. Rev. 1, 73 (1935).

[17] J. Eisenstaedt, Arch. Hist. Exact Sci. 2, 115 (1986).

[18] L. Bonolis, European Physical Journal H. 42, 311 (2017).

[19] E. Hubble, Proceedings of the National Academy of Sciences of the United States of America 3, 168 (1929).

[20] H. Dingle, Nature 139, 784 (1937).

[21] H. Dingle, Through Science to Philosophy (The Clarendon Press, Oxford, 1937).

[22] K. Hufbauer, en Reappraising Oppenheimer: Centennial Studies and Reflections, editado por C. Carson e D.A. Hollinger (Berkeley Papers in History of Science, California, 2005), v. 21.

[23] D. Kaiser, How the Hippies Saved Physics (W.W. Norton \& Company, New York, 2011).

[24] M. Fortun e S.S. Schweber, Social Studies of Science 4, 595 (1993).

[25] A.S. Blum, R. Lalli e J. Renn, Ann. Phys. 5, 344 (2016).

[26] S. Hawking e R. Penrose, Proc. Roy. Soc. Lond. A. 314, 529 (1970).

[27] R. Ruffini e J.A. Wheeler, Physics Today 4, 47 (2009). 